\title{
Estudio químico de la fracción de acetato de etilo del hongo Ganoderma lucidum cultivado en desechos agroindustriales
}

\author{
Rodrigo Daniel Salgado-Ordosgoitia ${ }^{1, *}$, Johanna Milena Arias-Martínez ${ }^{2}$, Amparo Del Cristo Acosta-Chávez ${ }^{3}$, \\ Jhonatan Andrés Rodríguez-Manrique ${ }^{1}$, Mario Frank Pérez-Pérez ${ }^{1}$ \\ ${ }^{1}$ Corporación Universitaria del Caribe (CECAR), Sincelejo, Sucre, Colombia \\ ${ }^{2}$ Institución Etno-educativa Afrocolombiana Cristo Rey, Puerto Escondido, Córdoba, Colombia \\ ${ }^{3}$ Institución Educativa Santa María Goretti, Montería, Córdoba, Colombia
}

\begin{abstract}
Resumen
Estudios en hongos han revelado que poseen un alto valor nutricional, comprobada acción farmacológica de los metabolitos secundarios producidos en sus cuerpos fructíferos, siendo muchas las especies estudiadas, destacando entre estas el hongo macromiceto Ganoderma lucidum, del cual se han aislado compuestos tipo terpenoides y sus derivados, de importancia biológica, mostrando efectividad en la disminución de la presión sanguínea, propiedades antinflamatorias, antivirales, hipoglicémicas e inmunoprotectoras. Por otro lado, el cultivo de hongos promueve la solución a problemas de tipo ambiental, ya que puede hacerse en desechos agroindustriales. El objetivo fue estudiar la composición química del hongo G. lucidum, cultivado en desechos agroindustriales y determinar diferencias entre los metabolitos obtenidos, con respecto a los de la especie del hongo en su estado silvestre. Para ello, se cultivó G. lucidum, en desechos agroindustriales y se estudió químicamente la fracción de acetato de etilo (AcOEt), mediante cromatografía de columna, cromatografía de capa fina, cromatografía en capa fina preparativa. Además de técnicas de análisis de estructuras CG-EM, RMN-1H, IR. Los resultados indicaron que la separación cromatografía, permitió la caracterización de 8 compuestos. De forma general, se puede concluir que el hongo cultivado, presenta una composición de metabolitos secundarios similar a la reportada en distintas investigaciones sobre la especie en su estado silvestre, estableciéndose que el compuesto mayoritario corresponde al ganoderiol B. (C) 2016. Acad. Colomb. Cienc. Ex. Fis. Nat.
\end{abstract}

Palabras clave: Ganoderma lucidum; Cultivo; Estudio químico; Metabolitos secundarios.

Chemical study of fraction ethyl acetate in the fungus Ganoderma lucidum, grown in agro-industrial waste.

\begin{abstract}
Studies in fungi have revealed that they have a high nutritional value, proven pharmacological action of the secondary metabolites produced in their fruiting bodies, being many species studied, emphasizing among these, the macromycete Ganoderma lucidum, from which have been isolated compounds type terpenoids and its derivatives, with biological significance, showing effectiveness in the lowering blood pressure, anti-inflammatory properties, antiviral, hypoglycemic and immunoprotective. On the other hand, mushroom cultivation promotes the solution to environmental problems, since it can be done in agro-industrial wastes. The objective was to study the chemical composition of the fungus G. lucidum, grown in agro-industrial wastes and to determine differences between the metabolites obtained, with respect to those of the species of the fungus in their wild state. To do so, G. lucidum, was cultivated in agro-industrial wastes and the ethyl acetate fraction was studied, throught chromatography column thin layer chromatography, preparative thin-layer chromatography. In addition, GC-MS, NMR-'H, IR analysis techniques were done. The results and preparative indicated that the chromatographic separation allowed the characterization of 8 compounds. In general, it can be concluded that cultivated fungus, presents a composition of secondary metabolites similar to the reported by various researchers about the species in its wild state, establishing the majority compound corresponding to the Ganoderiol B. (C) 2016. Acad. Colomb. Cienc. Ex. Fis. Nat.
\end{abstract}

Key words: Ganoderma lucidum; Cultivation; Chemical study; Secondary metabolites.

\section{Introducción}

El continuo deterioro de la salud y la contaminación ambiental han llevado a la búsqueda de nuevas fuentes de alimentos, drogas y agentes de control biológico, siendo el cultivo de los hongos y su estudio un prometedor mitigador en la solución de estos problemas. El alto valor nutricional, la comprobada acción farmacológica de los metabolitos secundarios producidos en sus cuerpos fructíferos y la reutilización que se le da a los desechos orgánicos, utilizados

\section{*Correspondencia:}

Rodrigo Daniel Salgado-Ordosgoitia, rodrigo.salgado@cecar.edu.co

Recibido: 05 de julio de 2016

Aceptado: 14 de septiembre de 2016 
como medio de cultivo (sustrato), son algunas de las bondades que ofrece el cultivo de las especies fúngicas (Suarez \& Nieto, 2013). Las rutas metabólicas de los hongos están determinadas por el sustrato de fijación, así hongos de una misma especie, desarrollados en diferentes sustratos o distintos lugares pueden variar en su composición química, aspectos relevantes para las empresas que incursionan en el cultivo de hongos con fines alimenticios y farmacéuticos (González, et al., 1999; You, et al., 2012).

En China la familia Ganodermataceae ha sido empleada por sus propiedades medicinales (Bishop, et al., 2015; Liu, et al., 2015). Algunas de sus especies: G. australe, $G$. applanatum, G. tsugae, G. labatum, G. sessile, G. colossum, G. curtisii, G. camosum y G. lucidum, han sido estudiadas químicamente encontrando dos clases principales de compuestos: triterpenos (Wang, et al., 2015) y polisacáridos, a los cuales se les atribuye actividad farmacológica (Wu, et al., 2013; Grienke, et al., 2011; Ríos, et al., 2012). Los triterpenos han mostrado efectividad en la disminución de la presión sanguínea, actúan como antiinflamatorios, antivirales, función hipolipemiante y hepatoprotectora. Los polisacáridos poseen efecto hipoglicémico e inmunopotenciador antitumoral (Ma, et al., 2015; Peng, et al., 2014; Cuéllar, et al., 1996; Baby, et al., 2015).

En la tabla 1S, http://www.raccefyn.co/index.php/ raccefyn/article/downloadSuppFile/391/1740, se muestran algunos metabolitos aislados del hongo Ganoderma lucidum en estado silvestre y cultivado.

\section{Materiales y métodos}

Cromatografía en capa fina (CCF). Placas de aluminio preparadas con sílica gel Merck ${ }^{\circledR}(20 \times 20 \mathrm{~cm})$, utilizando como eluyentes los sistemas Bencina: AcOEt y Hex: AcOEt, en diferentes concentraciones.

Cromatografía en columna (CC). Fase estacionaria Sílica gel tipo $60(0,063-0,200 \mathrm{~mm}$ de diámetro de la partícula, Merck®), utilizando como fase móvil Bencina: AcOEt y Hex:AcOEt en orden creciente de polaridad.

Cromatografía en capa fina preparativa (CCP). Cromatoplacas preparativas de Sílica gel $288.7 \mathrm{~K}, 254$, Merck ${ }^{\circledR}$ de 20 x $20 \mathrm{~cm}$ y 1,0 $\mathrm{mm}$ de espesor.

Cromatografía de gases acoplada a espectrometría de masas ( $\boldsymbol{C} \boldsymbol{G}-\boldsymbol{E M})$. Cromatógrafo de gases, marca HewlettPackard® modelo 6890 acoplado a un detector selectivo de masas Hewlett-Packard® 5973. Se utilizó una columna capilar HP 5 (30 m x 0,25 mm x 0,25 $\mu \mathrm{m})$ y Helio como gas de arrastre, a $10 \mathrm{ml} / \mathrm{min}$, temperatura de calentamiento $270^{\circ} \mathrm{C}$ hasta $310^{\circ} \mathrm{C}$, con una relación de split $1: 10$ y un volumen de inyección de muestra de 1 a $2 \mu \mathrm{L}$.

Espectro de resonancia magnética nuclear de hidrógeno (RMN-1H). Espectrógrafo marca Brucker® modelo AMX300 , operado a $300 \mathrm{MHz}$ para ${ }^{1} \mathrm{H}$, las muestras se disolvieron en cloroformo deuterado $\left(\mathrm{CDCl}_{3}\right)$ y como referencia interna se utilizó tetrametilsilano (TMS).

Espectro infrarrojo (IR). Espectrógrafo Perkin Elmer® FT-IR 1000
Reacciones de coloración. Reactivo de LiebermannBurchard modificado ( $80 \%$ de ácido acético, 16\% ácido sulfúrico, 4\% Agua), cuya reacción positiva produce coloraciones verdes, violetas, rojas entre otras.

Metodología general del cultivo del hongo

Producción de semilla comercial. El micelio de una cepa colombiana se transfiere a tubos de ensayo con los medios de cultivo: agar extracto de malta (EMA) y agar papa dextrosa (PDA). Del micelio se tomaron fragmentos de agar/micelio y se sembraron en cajas de petri y botellas con los mismos medios de cultivo. La producción de semilla comercial se realizó adicionando $500 \mathrm{~g}$ de trigo (lavado e hidratado, a una humedad final entre 40 y $45 \%$ ) en bolsas de polipropileno, posteriormente se esterilizaron en autoclave a $121^{\circ} \mathrm{C}$ durante 1 hora y se inocularon con fragmentos de agar/micelio obtenida en los frascos. Seguidamente fueron llevadas a incubación, esta etapa se realizó a 85\% de humedad relativa y finalizó después de 90 días cuando el micelio cubrió completamente el sustrato. Hasta que alcanzaran una completa invasión micelial.

Preparación del substrato. Se utilizó virutas y aserrín de eucalipto, bagazo de caña, cascarillas de cacao, café, coco, algodón, y salvado de trigo (en porcentajes equivalentes al $14,3 \%$ de cada desecho) carbonato de calcio y sulfato de calcio. Se empacó $1 \mathrm{Kg}$ de substrato en bolsas de polipropileno, se esterilizaron 2 horas.

Siembra del inóculo. Tasa de inoculación del 4\%, luego las bolsas de sustratos fueron selladas con un tapón de algodón revestido de gasa y ajustado con bandas de caucho.

Etapa de incubación y fructificación. El 85\% de humedad relativa y finalizó después de 90 días. La primera cosecha se realizó haciendo torsión en la base del pie o cortándolos, evitando residuos en el bloque que pueden inducir a la contaminación (Rodríguez, 2000; Varón, 2003)

Tratamiento del material fúngico. Ciento cincuenta gramos se sometieron a limpieza manual y lavado con agua, luego a secado en un horno con gradiente de temperatura creciente de 25 hasta $50^{\circ} \mathrm{C}$ por un periodo de 24 horas. Una vez secos se realizó molienda, el material se sometió a percolación (146,7 g) con etanol (EtOH) del 96\% (1 Litro). Este proceso se llevó a cabo en un frasco de color ámbar en la oscuridad, durante 28 días, después se filtró y el filtrado se concentró a presión reducida, obteniéndose el extracto etanólico crudo $(7,3 \mathrm{~g})$, que se sometió a partición con $\mathrm{H}_{2} \mathrm{O}$ : AcOEt (1:1). Tras separar la fase orgánica se secó con sulfato de sodio anhidro $\left(\mathrm{Na}_{2} \mathrm{SO}_{4}\right)$ y concentró a presión reducida, obteniéndose $7,195 \mathrm{~g}$ de extracto de la fracción de AcOEt.

\section{Resultados y discusión}

Características morfológicas del hongo Ganoderma lucidum obtenido del cultivo de la semilla comercial. Fructificación en forma de repisa semicircular adherido en forma sésil (lateralmente) al sustrato con un píleo dimidiado de color rojo ladrillo, de superficie seca, de consistencia 
dura, con bordes incurvados; los tamaños de estos hongos fueron variables en el cultivo como ha sido reportado por Guzmán, 1999 y Hernao, 1989. Estas características pueden ser observadas en la figura 1.

Análisis cromatográfico de la fracción de acetato de etilo. La fracción de AcOEt, se analizó por CCF, las mezclas de bencina:AcOEt y hexano:AcOEt mostraron la mejor resolución de los compuestos en las placas en orden creciente de polaridad, infiriéndose que el extracto de AcOEt, era una mezcla compleja de compuestos terpénicos y ácidos grasos. La separación de la mezcla se realizó por CC, eluyendo con gradiente discontinuo de polaridad creciente desde bencina hasta bencina: AcOEt 6:4, posteriormente hexano:AcOEt 5:5, hasta AcOEt y finalmente eluida con metanol $(\mathrm{MeOH})$. Mediante el monitoreo de la columna por CCF se agruparon aquellas fracciones que mostraron un comportamiento cromatográfico similar, obteniéndose las fracciones A-L, de las
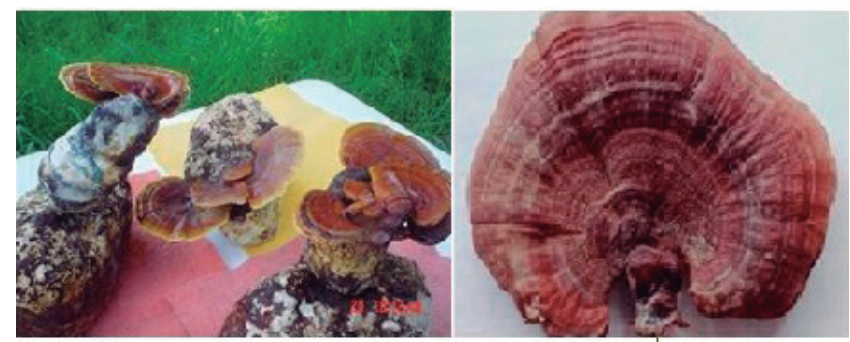

Figura 1. Fotografía de algunos bloques de cultivo y hongos producidos. cuales se seleccionaron para ser estudiadas las fracciones B, F e I, a su vez de estas fracciones se obtienen las subfracciones B8-1; B8-2; B8-3; B8-4; B8-5; F1; I4. Estas subfracciones son evaluadas por CG-EM, RMN ${ }^{1} \mathrm{H}$ e IR obteniéndose para cada una los siguientes datos espectrales que corresponden a los compuestos descritos en la tabla 1 .

\section{Compuestos obtenidos de la fracción $B$.}

B8-1. En el espectro de masas se observa un ion molecular a m/z $446\left(\mathrm{M}^{+}\right)$, que es consistente con la formula molecular $\mathrm{C}_{28} \mathrm{H}_{46} \mathrm{O}_{4}$ con un $\mathrm{IDH}=6$, cuyo número de carbonos es característico de un compuesto tetracíclico tipo ergostano, que por el cierre de estos anillos agota 4 insaturaciones restando 2 por ubicar. Los fragmentos $\mathrm{m} / \mathrm{z}$ $=428\left(\mathrm{M}-\mathrm{H}_{2} \mathrm{O}\right), 410\left(\mathrm{M}-2 \mathrm{H}_{2} \mathrm{O}\right), 303\left(\mathrm{M}-\mathrm{CL}-\mathrm{H}_{2} \mathrm{O}\right)$ y 207 (M-4 $\mathrm{H}_{2} \mathrm{O}-$-fisión $\left.\mathrm{D}\right)$, originadospor pérdidas sucesivas de moléculas de agua, permiten deducir la presencia de 4 grupos hidroxilos en la molécula.

El ion molecular a $\mathrm{m} / \mathrm{z}=385\left(\mathrm{M}^{+}-\mathrm{H}_{2} \mathrm{O}\right.$-Isop) que evidencia la presencia de un grupo isopropilo terminal en la cadena lateral. Al revisar la literatura, se encontró que los fragmentos de masas y el peso molecular, coinciden con los datos reportados por Yapita et al. (1999) y el compuesto se identificó como: 22E,24R-ergosta-7,22-dien-3 $3,5 \alpha, 6 \alpha, 9 \alpha-$ tetrol y en la figura 2 se representa su estructura.

B8-2. El ion molecular a $\mathrm{m} / \mathrm{z}=394$ es consistente con la formula molecular $\mathrm{C}_{28} \mathrm{H}_{42} \mathrm{O}$, cuyo $\mathrm{IDH}=8$, es decir, debido a la presencia de los anillos y cuatro dobles enlaces. La presencia de los iones a $\mathrm{m} / \mathrm{z}=376\left(\mathrm{M}^{+}-\mathrm{H}_{2} \mathrm{O}\right)$ y $\mathrm{m} / \mathrm{z}=$ $361\left(\mathrm{M}^{+}-\mathrm{H}_{2} \mathrm{O}-\mathrm{Me}\right)$ permiten inferir que se trata de un

Tabla 1. Sub-fracciones, fragmentos de masas y nombres de los compuestos obtenidos.

\begin{tabular}{|c|c|c|}
\hline Sub-Fracción & Señales espectroscópicas & Nombre del compuesto \\
\hline B8-1 & $\begin{array}{l}\text { Tiempo de retención 7,108min. Espectro de masas: iones m/z }(\%): 428\left(\mathrm{M}^{+}-2 ; 19,54\right) \text {, } \\
385(32,18), 303(16,09), 285(34,48), 267(10,34), 249(5,75), 69(100) .\end{array}$ & $\begin{array}{l}22 \mathrm{E}, 24 \mathrm{R} \text {-ergosta-7,22-dien- } 3 \beta, 5 \alpha, 6 \alpha, 9 \alpha- \\
\text { tetrol }\end{array}$ \\
\hline B8-2 & $\begin{array}{l}\text { Tiempo de retención 8,552 min. Espectro de masas: iones m/z (\%): } 394\left(\mathrm{M}^{+} ; 17,44\right) \text {, } \\
376(19,77), 361(6,39), 267(7,56), 251(100), 227(19,77), 209(26,74) .\end{array}$ & Ergosta-5,7,9,(11),22-tetraen-3-ol \\
\hline B8-3 & $\begin{array}{l}\text { Tiempo de retención 12,423 min. Espectro de masas: iones m/z (\%): } 392\left(\mathrm{M}^{+;} 18,39\right) \text {, } \\
377(9,19), 267(31,03), 266(100), 253(9,19), 225(4,02) .\end{array}$ & Ergosta-4,6,8(14),22-tetraen-3-ona \\
\hline B8-41 & $\begin{array}{l}\text { Tiempo de retención 12,941. Espectro de masas: iones m/z }(\%): 426\left(\mathrm{M}^{+}-2 ; 6,32\right) \text {, } \\
410(33,33), 395(22,99), 377(100), 285(27,59), 267(59,77), 207(52,87), 189(11,49) \text {, } \\
69(34,48)\end{array}$ & $\begin{array}{l}22 \mathrm{E}, 24 \mathrm{R}-5 \alpha, 6 \alpha \text {-epoxiergosta-8,22-dien- } \\
3 \beta, 7 \alpha \text {-diol, }\end{array}$ \\
\hline B8-42 & $\begin{array}{l}\text { Tiempo de retención } 14,242 \text { min Espectro de masas: iones m/z }(\%): 426\left(\mathrm{M}^{+}-2 \text {; }\right. \\
7,06), 410(65,88), 395(28,23), 377(100), 367(9,41), 285(38,82), 267(67,05), 207 \\
(44,70), 189(29,41), 69(53,53)\end{array}$ & 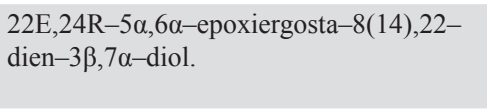 \\
\hline B8-5 & $\begin{array}{l}\text { Tiempo de retención } 13,512 \mathrm{~min} \text {. Espectro de masas: iones } \mathrm{m} / \mathrm{z}(\%): 428\left(\mathrm{M}^{+} ; 17,65\right) \text {, } \\
410(10,59), 385(38,82), 367(11,76), 316(5,88), 303(35,29), 301(100), 285(34,12) \text {, } \\
261(8,23), 243(22,35), 208(20,00), 175(21,18)\end{array}$ & Ergosta-6,22-dien-3 $\beta, 5 \alpha, 8 \alpha$-triol, \\
\hline \multirow[t]{3}{*}{ F1 } & $\begin{array}{l}\text { Tiempo de retención } 36,791 \mathrm{~min} \text {. Espectro de masas: iones } \mathrm{m} / \mathrm{z}(\%): 434\left(\mathrm{M}^{+} ; 10,46\right) \text {, } \\
419(8,14), 401(9,30), 325(100), 309(18,60) .\end{array}$ & \multirow{3}{*}{$\begin{array}{l}\text { 15 } \boldsymbol{\alpha}, 26,27-\text { trihidroxi-5 } \boldsymbol{\alpha}- \\
\text { lanosta-7,9(11),24-trien-3-ona } \\
\text { (Ganoderiol B). }\end{array}$} \\
\hline & $\begin{array}{l}\text { Espectro RMN-1'H: } \boldsymbol{\delta} \text { (integral, multiplicidad, } J \text {, asignación): } 0,69 \text { (3H, s, Me-18), 0,96 } \\
(3 \mathrm{H}, \mathrm{s}, \mathrm{Me}-30), 1,0(3 \mathrm{H}, \mathrm{s}, \mathrm{Me}-29), 1,14(3 \mathrm{H}, \mathrm{s}, \mathrm{Me}-28), 1,18(3 \mathrm{H}, \mathrm{s}, \mathrm{Me}-19), 0,94 \\
(3 \mathrm{H}, \mathrm{d}, 6,3, \mathrm{Me}-21), 5,43(1 \mathrm{H}, \mathrm{d}, \mathrm{H}-\mathrm{C} 11), 5,94(1 \mathrm{H}, \mathrm{d}, \mathrm{H}-\mathrm{C} 7), 5,57(1 \mathrm{H}, \mathrm{t}, \mathrm{H}-\mathrm{C} 24) \\
4,27(2 \mathrm{H}, \mathrm{s}, 2 \mathrm{H}-\mathrm{C} 26), 4,35(2 \mathrm{H}, \mathrm{s}, 2 \mathrm{H}-\mathrm{C} 27), 4,31(1 \mathrm{H}, \mathrm{dd}, \mathrm{H}-\mathrm{C} 15) .\end{array}$ & \\
\hline & $\begin{array}{l}\text { IR: } 3446,80 \mathrm{~cm}^{-1}, 2921,98 \mathrm{~cm}^{-1}, 1705,67 \mathrm{~cm}^{-1}, 1464,53 \mathrm{~cm}^{-1}, 1215-1320 \mathrm{~cm}^{-1} \text {, } \\
1010,63 \mathrm{~cm}^{-1}, 819,14 \mathrm{~cm}^{-1} \text {. }\end{array}$ & \\
\hline I4 & $\begin{array}{l}\text { Tiempo de retención 12,582 min. Espectro de masas: iones } \mathrm{m} / \mathrm{z}(\%): 279\left(\mathrm{M}^{+} ; 7\right), 167 \\
(27), 149(100), 123(1,2) .\end{array}$ & Dialquil ftalato \\
\hline
\end{tabular}


esterol tetracíclico tipo ergostano, hidroxilado en C3. La existencia de los picos a m/z $=251\left(\mathrm{M}^{+}-\mathrm{CL}-\mathrm{H}_{2} \mathrm{O}\right), \mathrm{m} / \mathrm{z}$ $=227\left(\mathrm{M}^{+}-\mathrm{CL}-\mathrm{D}-1 \mathrm{H}\right)$ y m/z $=209\left(\mathrm{M}^{+}-\mathrm{CL}-\mathrm{H}_{2} \mathrm{O}\right)$, cuyos fragmentos son característicos de núcleos $\Delta^{5,7,9,(11)}-3-$ Hidroxiandrostatrieno (Martínez, 2002), evidencian la presencia de tres instauraciones; mediante la revisión en la literatura, encontramos que el compuesto con dichas características expuestas anteriormente, corresponden al compuesto Ergosta-5,7,9,(11),22-tetraen-3-ol, lo cual se corroboró mediante el tipo de fragmentaciones y datos espectroscópicos reportados en bibliografía (Coy \& Nieto, 2009). En la figura 1S, hhttp://www.raccefyn.co/index.php/ raccefyn/article/downloadSuppFile/391/1741, se ilustra una representación de la estructura y el tipo de fragmentaciones del compuesto (B8-2) Ergosta-5,7,9,(11),22-tetraen-3-ol (Coy \& Nieto, 2009).

B8-3. El ion molecular a $\mathrm{m} / \mathrm{z}=392$ corresponde a la formula molecular $\mathrm{C}_{28} \mathrm{H}_{40} \mathrm{O}$, con un IDH $=9$, los iones $\mathrm{m} / \mathrm{z}=$ $377\left(\mathrm{M}^{+}-\mathrm{Me}\right), 267\left(\mathrm{M}^{+}-\mathrm{CL}\right), 266\left(\mathrm{M}^{+}-\mathrm{CL}-1 \mathrm{H}\right), 253\left(\mathrm{M}^{+}-\right.$ CL-Me), $225\left(\mathrm{M}^{+}-\mathrm{CL}-\mathrm{D}-1 \mathrm{H}\right)$, corroboran que se trata de una estructura típica de esteroles, donde el cierre de los anillos agota 4 instauraciones restando 5 por asignar. Dado que el patrón de fragmentación es similar al del Ergostano, permite inferir una instauración en la cadena lateral $\Delta^{22}$ (Martínez, 2002). Mediante revisión de la literatura se estableció que el compuesto presente es Ergosta-4,6,8(14),22-tetraen-3-ona (Zhao, et al., 2011) y su estructura se muestra en la figura 3.

B8-4. La presencia de un mismo compuesto se descarta por la diferencia en sus tiempos de retención de 12,941 y 14,242 $\min$ (B8-41 y B8-42 respectivamente), por lo que se presume que puede tratarse de compuestos epímeros o isómeros. En los espectros se pudo observar un ion a $\mathrm{m} / \mathrm{z}$ $=426(\mathrm{M}-2)$, característico de compuestos hidroxilados, por tanto la masa molecular de estos compuestos es 428 u.m.a. consistente con la formula molecular $\mathrm{C}_{28} \mathrm{H}_{44} \mathrm{O}_{3}$ la cual posee un IDH $=7$. Los picos observados a $\mathrm{m} / \mathrm{z}=285$ $\left(\mathrm{M}^{+}-\mathrm{H}_{2} \mathrm{O}-\mathrm{CL}\right), 267\left(\mathrm{M}^{+}-2 \mathrm{H}_{2} \mathrm{O}-\mathrm{CL}\right)$, en ambos espectros, infieren la existencia de por lo menos dos grupos hidroxilos, Se determinó que el peso molecular de la cadena lateral es de 125 u.m.a. correspondiente a la formula molecular $\mathrm{C}_{9} \mathrm{H}_{17}$ con $\mathrm{IDH}=1$, sugiriéndose una instauración $\Delta^{22}$, Además se observan los picos $\mathrm{m} / \mathrm{z}=410\left(\mathrm{M}^{+}-\mathrm{H}_{2} \mathrm{O}\right), 395$ $\left(\mathrm{M}^{+}-\mathrm{Me}-\mathrm{H}_{2} \mathrm{O}\right), 392\left(\mathrm{M}^{+}-2 \mathrm{H}_{2} \mathrm{O}\right), 377\left(\mathrm{M}^{+}-\mathrm{Me}-2 \mathrm{H}_{2} \mathrm{O}\right)$, que son coincidentes con los datos espectroscópicos reportados por Yue, et al. (2001) y Zhou, et al. (2011), que pueden ser asignados indistintamente a los siguientes compuestos: 22E, $24 \mathrm{R}-5 \alpha, 6 \alpha$-epoxi-ergosta-8,22-dien-3 $\beta, 7 \alpha$-diol y 22E, 24R-5 $\alpha, 6 \alpha$-epoxi-ergosta-8(14),22-dien-3 $\beta, 7 \alpha$-diol. En la figura 4, se ilustran las estructuras de los compuestos (B8-41) y (B8-42) 22E,24R-5 $\alpha, 6 \alpha-$-epoxi-ergosta-8,22dien-3 $3,7 \alpha$-diol y 22E,24R-5 $\alpha, 6 \alpha$-epoxi-ergosta- $8(14), 22-$ dien-3 $3,7 \alpha$-diol.

B8-5. Los fragmentos generados por pérdidas sucesivas de tres moléculas de agua (428 a 410, 385 a 367 y 367 a $349)$, y por la presencia de los fragmentos $\mathrm{m} / \mathrm{z}=285\left(\mathrm{M}^{+}-\right.$

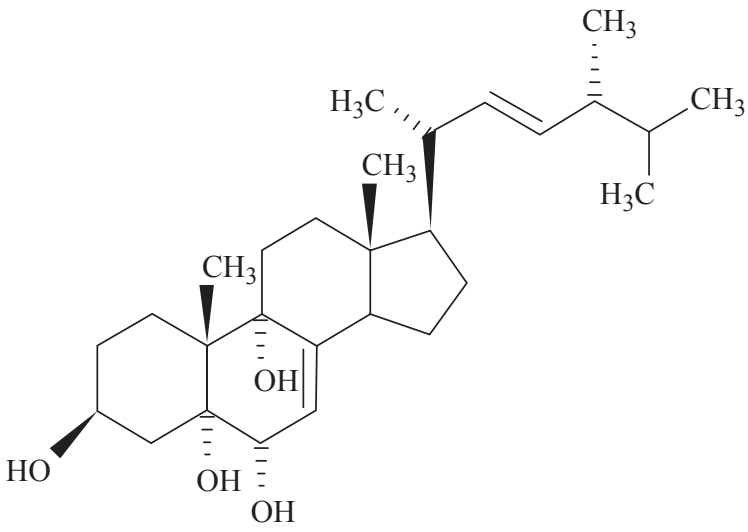

Figura 2. Estructura del compuesto (B8-1) 22E,24R-ergosta7,22-dien-3 $\beta, 5 \alpha, 6 \alpha, 9 \alpha$ tetrol.

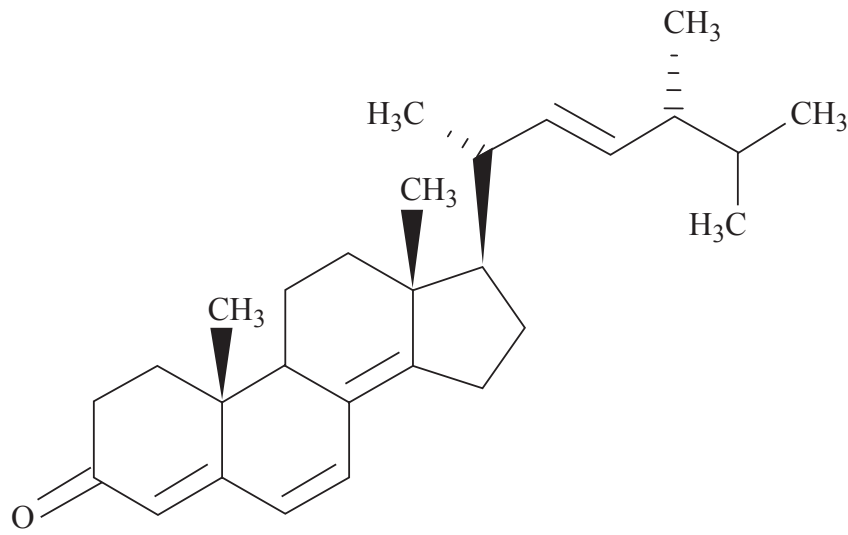

Figura 3. Estructura del compuesto (B8-3) Ergosta-4,6, 8(14), 22-tetraen-3-ona.

$\left.\mathrm{H}_{2} \mathrm{O}-\mathrm{CL}-2 \mathrm{H}\right), 267\left(\mathrm{M}^{+}-2 \mathrm{H}_{2} \mathrm{O}-\mathrm{CL}-2 \mathrm{H}\right)$, y $249\left(\mathrm{M}^{+}-3 \mathrm{H}_{2} \mathrm{O}-\right.$ CL-2H), permiten establecer que la molécula posee de tres grupos hidroxilos.

Esta sustancia tiene un peso molecular de 430 u.m.a. consistente con la formula molecular $\mathrm{C}_{28} \mathrm{H}_{46} \mathrm{O}_{3}$ con IDH $=6$ $\mathrm{y}$ es el ion molecular en el espectro de masas, pero con una baja abundancia relativa, lo que indicaría que el fragmento a $\mathrm{m} / \mathrm{z}=428$ corresponde al $\mathrm{M}^{+}-2 \mathrm{H}$, pérdida de común ocurrencia en espectros de sustancias de esta clase.

Revisando la literatura, se encontró que los datos espectroscópicos son consistentes con los reportados por Gonzales et al. (1983) para el compuesto Ergosta-6,22dien-3 $\beta, 5 \alpha, 8 \alpha$-triol, quienes además describen las fragmentaciones características para este compuesto. En la figura $2 \mathrm{~S}$, http://www.raccefyn.co/index.php/raccefyn/article/download SuppFile/391/1742, se ilustra la estructura y el esquema de fragmentaciones del compuesto (B8-5) Ergosta-6,22-dien$3 \beta, 5 \alpha, 8 \alpha-$ triol.

Compuesto obtenido de la fracción $F$. En el espectro IR (Figura 5) se observan las siguientes bandas: 3446, $80 \mathrm{~cm}^{-1}$ tensión del grupo hidroxilo y flexión a $1010,63 \mathrm{~cm}^{-1}$, 


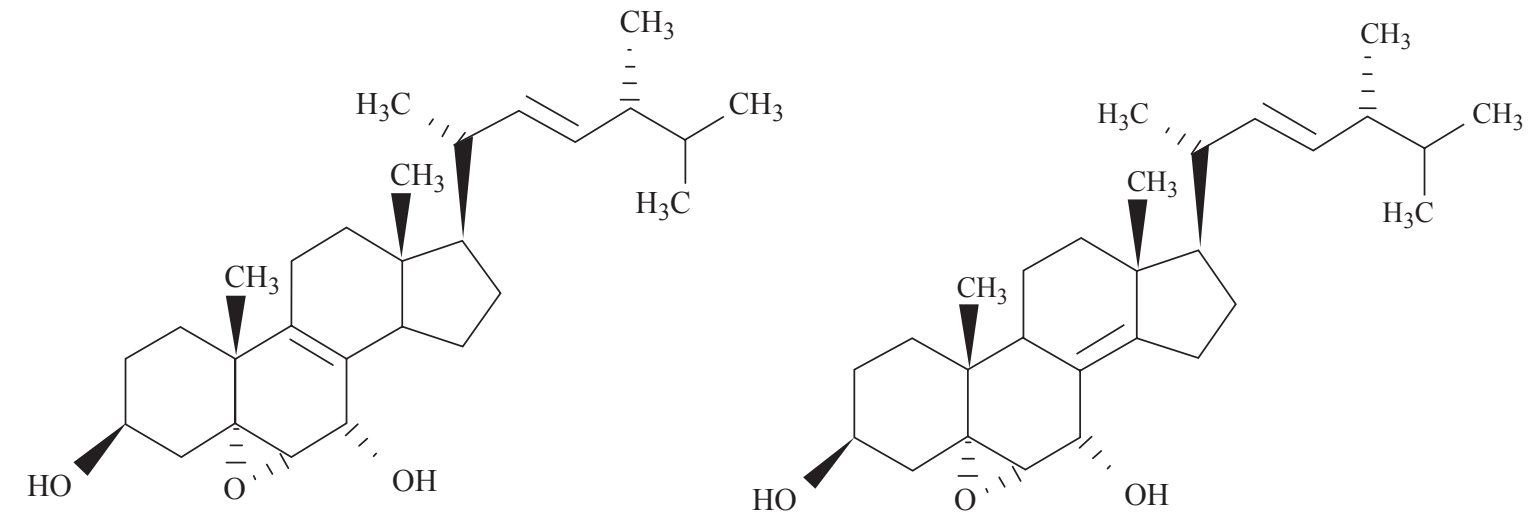

Figura 4. Estructuras de los compuestos (B8-41) y (B8-42) 22E,24R-5 $\alpha, 6 \alpha$-epoxi-ergosta-8,22-dien-33,7 $\alpha$-diol y 22E,24R-5 $\alpha, 6 \alpha-$ epoxiergosta-8(14),22-dien-3 $\beta, 7 \alpha$-diol.

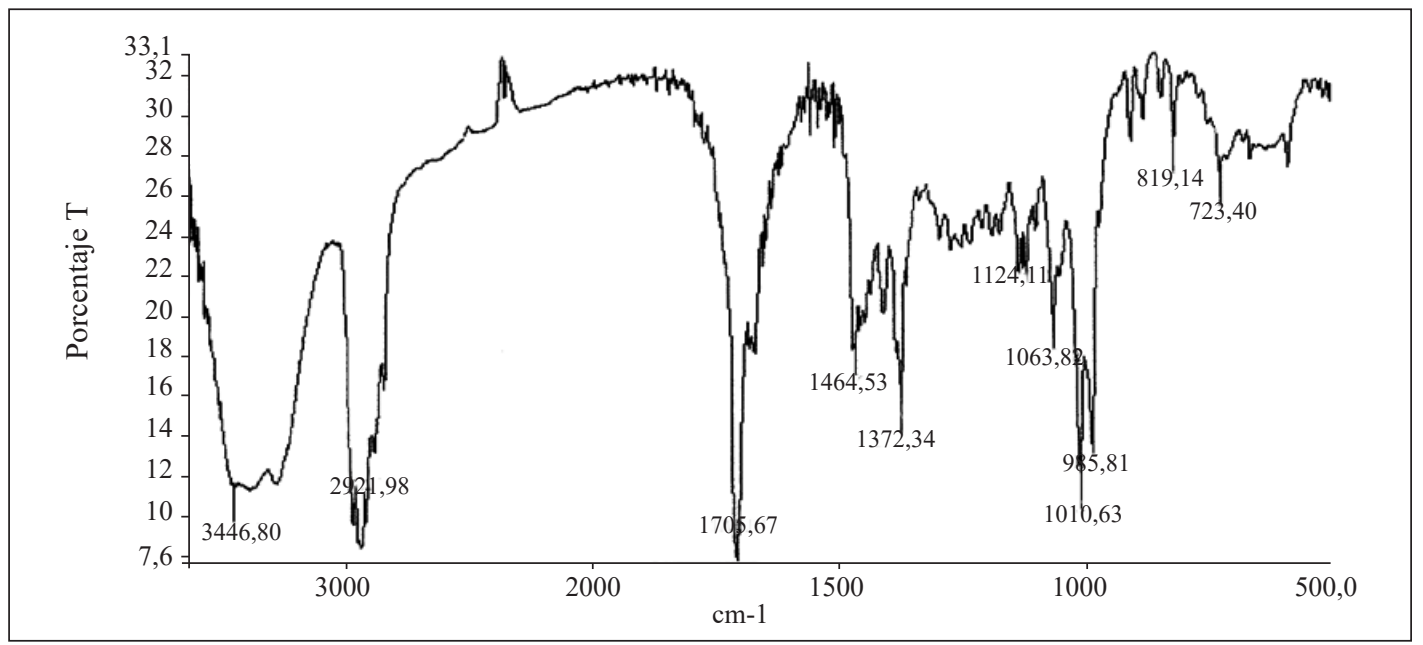

Figura 5. Espectro de IR 15a,26,27-trihidroxi-5a-lanosta-7,9(11),24-trien-3-ona.

banda fuerte en 1705,67 $\mathrm{cm}^{-1}$, debido a la tensión del grupo carbonilo y las de flexión en el intervalo de 1215-1320 $\mathrm{cm}^{-}$ 1, la banda a $2921,98 \mathrm{~cm}^{-1}$, por la tensión de los metilos y metilenos y las de flexión de estos a $1464,53 \mathrm{~cm}^{-1}$, por último la banda presente a $819,14 \mathrm{~cm}^{-1}$ por la tensión de los metilos.

En el espectro RMN-1 $\mathrm{H}$ (Figura 6) presenta singletes y dobletes entre 0 y 1,2 ppm originados por grupos metílicos que pueden relacionarse con un compuesto tipo lanostano; entre 1,3 y 2,8 ppm se observa un conjunto de señales complejas debidas a los protones metilénicos y metínicos, así como señales características de protones vinílicos entre 5,0 y 6,0 ppm. A campo alto se distingue una señal a $\delta 0,69$ con multiplicidad de singlete, que integra para tres protones que se asigna al Me-18 a $\delta 0,96$ y 1,00, se observan dos singletes, que integra para seis protones que corresponden a los $\mathrm{Me}-$ 30 y Me-29, también se distinguen dos singletes mas a $\delta$ 1,14 para el Me-28 y $\delta 1,18$ para el Me-19. La señal que se encuentra a $\delta 0,94$ con multiplicidad de doblete $(\mathrm{j}=6,3 \mathrm{~Hz})$, se asignó al Me-21. De acuerdo con datos bibliográficos (Shiao, et al., 1988; Arisawa, et al., 1986; Arisawa, et al.,1988; Morigiwa, et al., 1986; González, et al., 2002), las señales en $\delta 5,43,5,94$ y 5,57, que integran para un protón cada una, se asignan respectivamente a los protones vinílicos (H-C11), (H-C7) y (H-C24) de un núcleo Lanosta-7,9,(11) -dieno; con cadena lateral insaturada en el C24.

En el espectro no se observaron señales en $\delta 3,1-3,3$ que son característica del protón del grupo hidroxilo cuando está en posición 3, lo que indica que el núcleo lanostano no se encuentra hidroxilado en dicha posición y en la cual es posible asignar un grupo carbonilo, corroborado en el espectro de IR.

Las señales con desplazamientos químicos y multiplicidad de singletes, que integran para dos protones cada una, observados a campos bajos en $\delta 4,27$ y 4,35, corresponden a protones desprotegidos por la presencia de dobles enlaces carbono-carbono y un heteroátomo, como el oxígeno del grupo funcional hidroxilo, se asignan a los protones metilénicos (2H-C26) y (2H-C27) (Gutiérrez, et al., 1994; Guzmán, 1999; Hernao, 1989; Yapita, et al., 1999; Nieto, et al., 2001) terminales de un sistema de cadena lateral dihidroxilada en dichas posiciones. 
La señal que se observa a $\delta 4,31$, con multiplicidad de doble doblete puede ser inferida como un protón geminal, a un hidroxilo $\alpha$ en un anillo de 5 miembros, deduciéndose la existencia de un grupo hidroxilo en posición 15, lo cual no es inusual puesto que se conocen ejemplos; como el

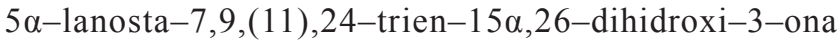
(González, et al., 2002) y el Ácido-3 $\beta, 15 \alpha$-dihidrixilanosta8,24-dien-26-oico (Shiao, et al., 1988).

De este análisis se dedujo que la molécula posee un total de 3 grupos hidroxilos, dos en la cadena lateral y uno en el anillo de cinco miembros.

En el espectro de masas (Figura 7), se observa un ión molecular a $\mathrm{m} / \mathrm{z}=434$, originado por una doble deshidratación de la molécula, puesto que esta es una de las características más frecuentes de los espectros de masas de alcoholes. El peso molecular del compuesto es $\mathrm{M}^{+}=470$ u.m.a., correspondiente a la suma del ion molecular a $\mathrm{m} / \mathrm{z}=$ 434, más las dos moléculas de agua perdidas y es consistente con la formula molecular $\mathrm{C}_{30} \mathrm{H}_{46} \mathrm{O}_{4}$, con un IDH de 8, el cual corresponde a cuatro anillos y cuatro dobles enlaces.
Se observan los iones $\mathrm{m} / \mathrm{z}=419\left(\mathrm{M}^{+}-2 \mathrm{H}_{2} \mathrm{O}-\right.$-metilo $)$, $\mathrm{m} / \mathrm{z}=401\left(\mathrm{M}^{+}-3 \mathrm{H}_{2} \mathrm{O}-\right.$ metilo$)$, corroborándose la presencia de tres moléculas de agua en el compuesto, $\mathrm{m} / \mathrm{z}=325\left(\mathrm{M}^{+}-\right.$ $\mathrm{CL}-2 \mathrm{H}), \mathrm{m} / \mathrm{z}=309\left(\mathrm{M}^{+}-\mathrm{CL}-\mathrm{H}_{2} \mathrm{O}\right)$, característico cuando en los anillos existe un grupo hidroxilo.

La cadena lateral corresponde a 143 u.m.a. $\left(\mathrm{M}^{+}-327\right)$, consistente con la formula molecular $\mathrm{C}_{8} \mathrm{H}_{15} \mathrm{O}_{2}$ y con IDH = 1 , el compuesto corresponde a $15 \alpha, 26,27$-trihidroxi-5 $\alpha-$ lanosta-7,9(11),24-trien-3-ona (Ganoderiol B) y se muestran las siguientes fragmentaciones. En la figura 3S, http:// www.raccefyn.co/index.php/raccefyn/article/download SuppFile/391/1743, se ilustra una representación de la estructura y el esquema de fragmentaciones del compuesto (F1) 15 $\alpha, 26,27$-trihidroxi-5 $\alpha$-lanosta-7,9(11),24-trien-3-ona.

Compuesto obtenido de la fracción I. Presenta un patrón de fragmentaciones típico de ftalatos, se podría pensar que se trata de impurezas de los solventes, pero no se descarta la posibilidad de que se traten de un metabolito secundario del hongo, ya que se han encontrado dialquil ftalatos formando parte dentro de la composición de varias especies del mismo

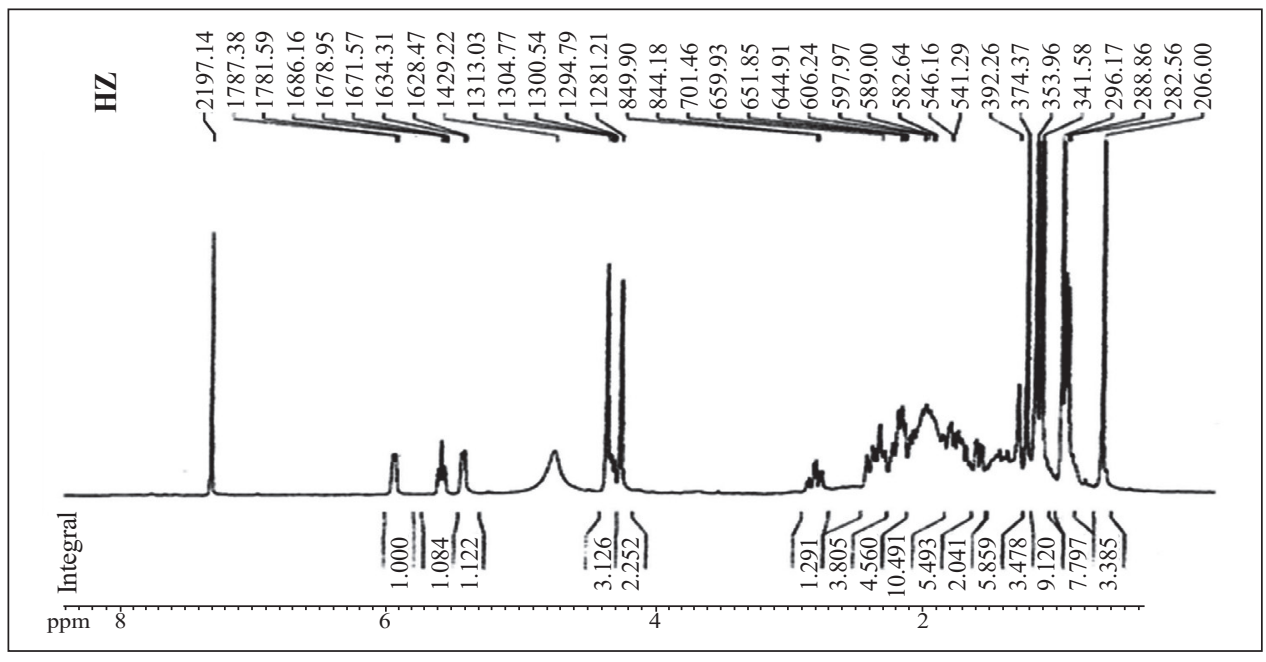

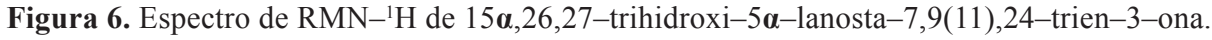

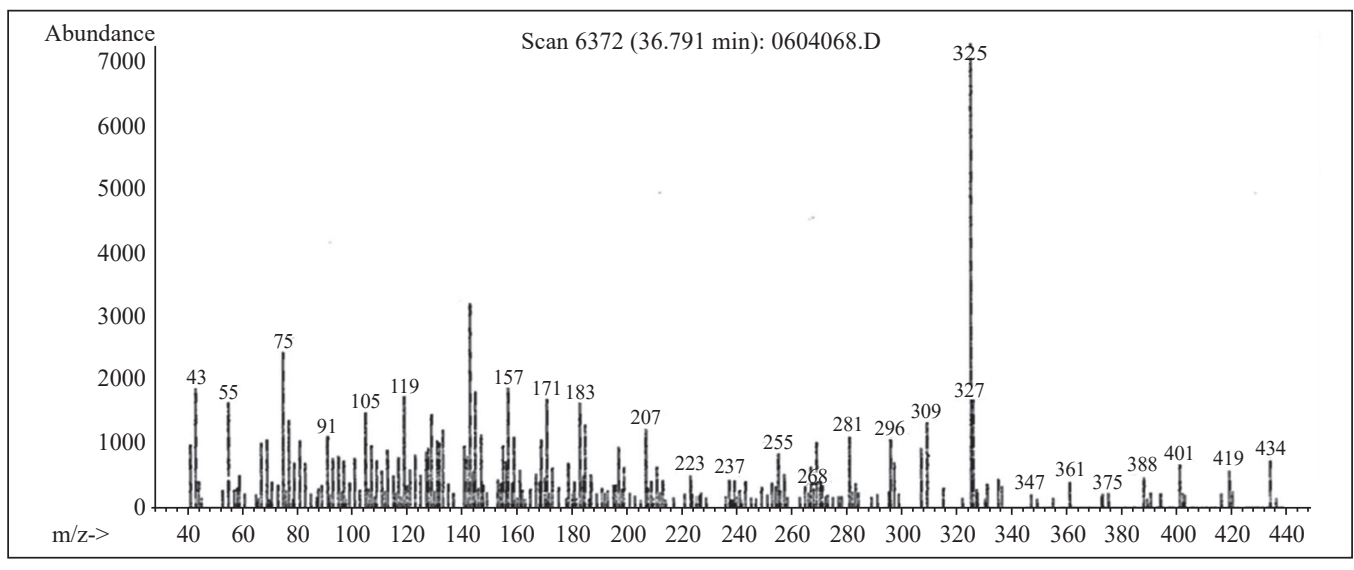

Figura 7. Espectro de masa del compuesto 15 $2,26,27$-trihidroxi-5 $\alpha$-lanosta-7,9(11),24-trien-3-ona. 
género (Gutiérrez, et al., 1994). Y se observan en el siguiente modelo de fragmentos. En la figura 4S, http://www.raccefyn. co/index.php/raccefyn/article/downloadSuppFile/391/1744, se representa la estructura y modelo de fragmentaciones del compuesto (I4) Dialquil ftalato.

\section{Conclusiones}

Del hongo G. lucidum obtenido del cultivo de la semilla comercial se identificaron compuestos tipo ergostano como son: 22E,24R-ergosta-7,22-dien-3 $\beta, 5 \alpha, 6 \alpha, 9 \alpha$-tetrol; ergosta$5,7,9,(11), 22$-tetraen-3-ol; ergosta-4,6,8(14),22-tetraen-3ona; 22E, 24R-5 $\alpha, 6 \alpha$-epoxiergosta- 8 ; 22-dien-3 $\beta, 7 \alpha$-diol;

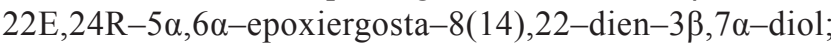
ergosta-6,22-dien-3 $\beta, 5 \alpha, 8 \alpha$-triol; uno tipo lanostano $15 \alpha, 26$, 27-trihidroxi-5a-lanosta-7,9(11),24-trien-3-ona; además del dialquil ftalato.

El hongo G. lucidum cultivado, presenta una composición de metabolitos secundarios similar a los encontrados y que han sido reportados en distintas investigaciones sobre la especie recolectada en su estado silvestre.

El compuesto mayoritario fue el ganoderiol B, previamente extraído del carpoforo de la especie silvestre y cuya principal característica estructural es la de un derivado oxigenado del lanostano, con 30 átomos de carbono y tres dobles enlaces en C9, C11 y C24; y con oxigenación en el C3, C15, C26 y C27, estas características estructurales han mostrado actividad biológica en la inhibición de la síntesis del colesterol (hipocolesterolemicas), hipotensiva, hepatoprotectora y antivirales. Así mismo, un grado mayor de oxigenación de las funciones alcohólicas (derivados ácidos y acetilados) aumenta la actividad citotóxica.

\section{Información suplementaria}

Tabla 1S. Metabolitos aislados del hongo Ganoderma Lucidum silvestre y cultivado. Vea la tabla $1 \mathrm{~S}$ en: http:/www.raccefyn.co/ index.php/raccefyn/article/downloadSuppFile/391/1740

Figura 1S. Estructura y esquema de fragmentaciones del compuesto (B8-2) Ergosta-5,7,9,(11),22-tetraen-3-ol. Vea la figura 1S en: http://www.raccefyn.co/index.php/raccefyn/article/download SuppFile/391/1741

Figura 2S. Estructura y esquema de fragmentaciones del compuesto (B8-5) Ergosta-6,22-dien-3 $\beta, 5 \alpha, 8 \alpha$-triol. Vea la figura $2 \mathrm{~S}$ en: http://www.raccefyn.co/index.php/raccefyn/article/download SuppFile/391/1742

Figura 3S. Estructura y esquema de fragmentaciones del compuesto del compuesto (F1) 15 $\alpha, 26,27$-trihidroxi-5 $\alpha$-lanosta-7,9(11),24trien-3-ona. Vea la figura 3S en: http://www.raccefyn.co/index. php/raccefyn/article/downloadSuppFile/391/1743

Figura 4S. Estructura y esquema de fragmentaciones del compuesto (I4) Dialquil ftalato. Vea la figura 4S en: http://www.raccefyn.co/ index.php/raccefyn/article/downloadSuppFile/391/1744

\section{Agradecimientos}

A la Corporación Universitaria del Caribe CECAR, Institución que financia las labores investigativas de los docentes Rodrigo Salgado, Jhonatan Rodriguez, Mario Pérez.

A la Universidad de Córdoba, por permitir la realización de esta investigación en sus instalaciones del laboratorio de productos naturales, aportando el uso de equipos y reactivos.

A la Incubadora de empresas de base tecnológica de Antioquia (IEBTA) y a la empresa pre-incubadora Hongos nutraceuticos, quienes suministraron la cepa nativa del hongo y financiaron parte de los análisis espectroscópicos.

Al Departamento Administrativo de Ciencia, Tecnología e Innovación (Colciencias), entidad que financia los estudios de maestría de Jhonatan Rodriguez.

\section{Conflicto de intereses}

Los autores declaran no tener conflicto de intereses.

\section{Referencias}

Arisawa, M., Fujita, A., Saga, M., Fukumura, H., Hayashi, T., Shimizu, Mineo., et al. (1986). Three New Lanostanoids from Ganoderma lucidum. J. Nat. Prod. 49 (4): 621-625.

Arisawa, M., Fujita, Akio., Hayashi, T., Shimizu, M., Morita, N., Kikuchi, T., et al. (1988). Revision of 1H- and 13C-nmr Assignments of Lanostanoids from Ganoderma lucidum by 2D-nmr Studies. J. Nat. Prod. 51 (1): 54-59.

Baby, S., Johnson, A. J., Govindan, B. (2015). Secondary metabolites from Ganoderma. Phytochemistry. 1: 66-101.

Bishop, K. S., Kao Chi, H. J., Yuanye, Xu., Glucina, M. P., Paterson R. R. M., Ferguson, L. R. (2015). From 2000 years of Ganoderma lucidum to recent developments. Phytochemistry. 1: 56-65.

Coy, E., Nieto, I. J. (2009). Sterol composition of the macromycte. Chem. Nat. Compd. 1: 193-196.

Cuéllar, M. J., Giner, R. M., Recio, M. C., Just, M. J., Máñez, S., Ríos J. L. (1996). Two Fungal Lanostane Derivatives as Phospholipase A2 Inhibitors. J Nat Prod. 1: 977-979.

González, A. G., León, F., Rivera, A., Padrón, J. I., González Plata, J., Zuluaga, J. C., et al. (2002). New Lanostanoids from the Fungus Ganoderma concinna. J. Nat. Prod. 65 (3): 417-21.

González, A., Bermejo, J., Toledo, F. (1983). The steroids and fatty acids of the basidiomycete Scleroderma polyrhizum. Phytochemistry. 22 (4): 1049-1050.

González, A., León, F., Rivera, A., Muñoz, C. M., Bermejo, J. (1999). Lanostanoid Triterpenes from Ganoderma lucidum. J. Nat. Prod. 1: 1700-1701.

Grienke, U., Mihály-Bison, J., Schuster, D., Afonyushkin, T., Binder, M., Guan, S-h., Cheng, C-r, Wolber, G., Stuppner, H., Guo, D-a, Valery N. Bochkov, V. N., Rollinger, J. M. (2011). Pharmacophore-based discovery of FXR-agonists. Part II: Identification of bioactive triterpenes from Ganoderma lucidum. Bioorganic \& Medicinal Chemistry. 19 (22): 6779-6791.

Gutiérrez, A., Caramelo, L., Prieto, A., Martínez, M. J., Martínez, A. T. (1994). Anisaldehyde production and arylalcohol oxidase and dehydrogenase activities in ligninolytic fungi of the genus Pleurotus. Applied and Environmental Microbiology. 60 (6): 1783-1788. 
Guzmán, G. (1999). Análisis cualitativo y cuantitativo de la diversidad de los hongos de México. La Diversidad Biológica de Iberoamerica Vol II. Acta Zoológica Mexicana. 1: $111-175$.

Hernao, L. (1989). Notas sobre Afiloforales colombianos. Rev. Caldasia. 1: 23-36.

Lin, L-J., Shiao, M-S. (1988). Seven new triterpenes from Ganoderma lucidum. J. Nat. Prod. 1: 918-924.

Liu, C., Yang, N., Song, Y., Wang, L., Zi, J., Zhang, S., Dunkin, D., Busse, P., Weir, D., Tversky, J., Miller, RL., Goldfarb, J., Zhan, J., Li XM. (2015). Ganoderic acid $\mathrm{C} 1$ isolated from the anti-asthma formula, ASHMI ${ }^{\mathrm{TM}}$ suppresses TNF- $\alpha$ production by mouse macrophages and peripheral blood mononuclear cells from asthma patients. International Immunopharmacology. 27 (2): 224-231.

Ma, H-T., Hsieh, J-F., Chen, S-T. (2015). Anti-diabetic effects of Ganoderma lucidum. Phytochemistry. 1: 109-113.

Manavalan, T., Manavalan, A., Thangavelu, K. P., Heese K. (2012). Secretome analysisof Ganoderma lucidum cultivated in sugarcane bagasse. J. Nat. Prod. 1: 298-309.

Martínez, Alejandro. (2002). Esteroles. Universidad de Antioquia; Facultad de Química Farmacéutica. Medellin - Colombia.

Masao, H., Isao, A., Chieko, I., Tsutomu, F., Motoo, S. (1987). Ganoderic acid derivatives and ergosta-4,7,22-triene3,6-dione from Ganoderma lucidum. Phytochemistry. 1: 2797-2803.

Moreno, H., Martínez, A., Fujimoto, Y. (2011). Aislamiento e identificación de dos esteroles y un triterpenoide del cuerpo fructífero de Ganoderma lucidum cultivado en Colombia. Vitae. 1: 11-15.

Morigiwa, A., Kitabatake, K., Fujimoto, Y., Ikekawa, N. (1986). Angiotensin converting enzyme-inhibitory triterpenes from Ganoderma lucidum. Chem Pharm Bull. 34 (7): 3025-3028.

Nieto, I. J., Valencia, M. A. (2001). Esteroles, Ácidos Grasos e Hidrocarburos de Los Cuerpos Fructíferos De Ganoderma australe. Bol. Soc. Chil. Quím. 47 (4): 511-516.

Peng, X-R., Liu, J-Q., Wang, C-F., Li, X-Y., Shu, Y., Zhou, L., et al. (2014). Hepatoprotective Effects of Triterpenoids from Ganoderma cochlear. J. Nat. Prod. 1: 737-743.

Ríos, J-L., Andújar, I., Recio, M-C., Giner, R-M. (2012). Lanostanoids from Fungi: A Group of Potential Anticancer Compounds. J. Nat. Prod. 75 (11): 2016-2044.
Rodríguez, V. N. (2000). Investigación básica sobre el cultivo de hongos tropicales en residuos agroindustriales de la zona cafetera colombiana. Chinchiná, Cenicafé (experimento QIN-6-01), disciplina de Química Industrial, p. 90.

Shiao, M-S., Lin, L-J., Yeh, S-F. (1988). Triterpenes from Ganoderma lucidum. Phytochemistry. 1: 2911-2914.

Suárez, C., Nieto, I. (2013). Cultivo biotecnológico de macrohongos comestibles: una alternativa en la en la obtención de Nutracéuticos. Revista Iberoamericana de Micología. 30 (1): $1-8$.

Varón, M. (2003). Cultivo de hongos tropicales agroindustriales del Departamento del Tolima (Tesis de pregrado). Universidad del Tolima, Facultad de Ciencias Básicas. Tolima-Colombia.

Wang, K., Bao, L., Xiong, W., Ma, K., Han, J., Wang, W., Yin, W., Liu, H. (2015). Lanostane Triterpenes from the Tibetan Medicinal Mushroom Ganoderma leucocontextum and Their Inhibitory Effects on HMG-CoA Reductase and a-Glucosidase, J. Nat. Prod. 78 (8): 1977-1989.

Wu, GS., Guo, JJ., Bao, JL., Li, XW., Chen, XP., Lu, JJ., Wang YT. (2013). Anti-cancer properties of triterpenoids isolated from Ganoderma lucidum - a review. Expert Opin Investig Drugs. 22 (8): 981-92.

Yapita, Y., Endo, M., Tani, Y., Machida, K., Amemiya, K., Furumura, K., et al. (1999). Sterol Constituents from Seven Mushrooms. Chem. Pharm. Bull. 47 (6): 847- 851.

You, B-J., Chang, W-T., Chung, K-R., Kuo, Y-H., Yang, C-S., Tien, Ni., et al. (2012). Effect of solid-medium coupled with reactive oxygen species on ganoderic acid biosynthesis and MAP kinase phosphorylation in Ganoderma lucidum. Food Res. Int, 1: 634-640.

Yue, J-M., Chen, S.N., Lin, Z-W., Sun, H-D. (2001). Sterols from the fungus Lactarium volemus. Phytochemistry. 1: 801-806.

Zhao, Y-Y., Zhang, L., Mao, J-R., Cheng, X-H., Lin, R-C., Zhang, Y., et al. (2011). Ergosta-4,6,8(14),22-tetraen3 -one isolated from Polyporus umbellatus prevents early renal injury in aristolochic acid-induced nephropathy rats. J. Pharm. Pharmacol. 1: 1581-1586.

Zhou, Z.Y, Tan, J.W y Liu, J.K. (2011). Two new polyols and a new phenylpropanoid glycoside from the basidiomycete Lactarius deliciosus. Fitoterapia. 82: 1309-1312. 